\title{
Gender Prediction of Consumers Using Offline Purchase Data
}

\author{
Cong Wang, Yang Ji \\ School of Information and Communication Engineering, BUPT, Beijing, China \\ e-mail: merrier1993@163.com, e-mail: jiyang@bupt.edu.cn
}

Keywords: gender prediction, performance study, offline purchase data

\begin{abstract}
Demographic attributes such as gender of consumers provide important in-formation for marketing, personalization, and user behavior research. With the growing necessity for gender information in personalized intelligent systems, gender prediction of consumers has become an important research issue. This paper addresses the problem of predicting consumers' gender based on purchase data and some external factors, such as weather and name. According to the characteristics of offline data, we compared the performance of different algorithms and the contribution of different features to gender prediction was compared. From the experiments conducted on real-world datasets, we found the most important features and the best performing algorithms that influenced the gender prediction of offline purchase da-ta. This study provided suggestions for apparel offline markets to develop effective marketing strategies to reach their target market, for consumer educators and for educators in the retail merchandizing area to prepare their students for future careers.
\end{abstract}

\section{Introduction}

In today's digital world, customers can engage in business transactions to purchase products or services over the Web twenty-four hours a day, seven days a week. We call this phenomenon on-line shopping and it has been particularly popular since the mid-1990s. Ever since, on-line shop-ping has been of interest for numerous researches. Gender is an important factor affecting consumers' online shopping behaviors and re-search has indicated that gender is a discriminating factor in the online information searches [1], shopping preferences [2], the effect of other consumer's reviews [3], merchandise quality and interaction quality [4] and so on; However, most researchers focused on the gender classification by theirs online shopping behaviors, few papers have explored how to classify men and women only by theirs offline purchase data.

In practice, it is usually not easy to analysis consumers' demographic attributes only by theirs offline behaviors because researchers can't obtain sufficient behavior information. The difficulty in classifying men and women in retail scenario raises an interesting research question: Can we inference users' gender automatically only based on their purchase behaviors and other known external factors? Although some recent studies suggest that gender attributes are predictable from different behavioral data, such as linguistics writing $[5,6,7,8]$, facial images $[9,10,11]$, social media $[12,13,14]$ and mobile data [15] to our best knowledge, seldom practice has been conducted on purchase behaviors in retail scenario.

Shelja J. Kuruvilla, Nishank Joshi and Nidhi Shah [16] explored mall-shopping habits in India and attempted to identify and contrast possible differences between genders. They found that in India there are significant differences in shopping behavior that can be ascribed to gender. Pengfei Wang, Jiafeng Guo and Yanyan Lan et al. [17] proposed a mod-el to automatically learn the representations from users' purchase data for predicting five demographic attributes (gender, marital status, income, age, and education level) simultaneous. However, collecting users' demo-graphic information mostly is a particularly difficult problem for traditional offline retailers. Qiufang Peng and Yang Liu [18] analyzed the difference between online shopping and entity store shopping: first, there are more women than men in online shopping while women prefer to shop offline; second, if it is to buy some 
valuable goods, customers prefer going to the store rather than shopping online.

Motivated by the above remarks, we believe that consumer behavior in online and traditional supermarkets is different in some respects. It's a formidable task to classify men and women only by offline purchase data because of the lack of information. To address this problem, we propose a model to predict gender from users' purchase data, which includes transaction time, brand, category and some other available transaction information. This research will contribute to the body of consumer behavior literature by identifying consumers' shopping orientations and gender differences and their influences on offline shopping behaviors.

The rest of this paper is being organized as follows: Section 2 describes about the Literature Survey on gender differences on shopping behaviors and gender prediction. Section 3 describes experiment data and methods. In section 4 we present our proposed model in detail and gives the Experimental Results. Finally, we summarize the main insights from our study and suggest further research opportunities in section 6 .

\section{Literature Survey}

In this section, we briefly review three research areas related to our work: differences between online and offline, gender differences and gender prediction.

\subsection{Differences Between Online and Offline}

There is increasing interest in determining whether there are systematic differences in consumer choice behavior between online and offline stores. An issue of particular interest to both practitioners and academics is in understanding the reasons for these differences if they exist. Few papers have explored how consumer behavior online differs from consumer behavior offline. Exceptions are a conceptual paper by Arvind and Wu [19] and an experimental study by Peter, Wilson and Davis [20].

Arvind and $\mathrm{Wu}$ [19] point out that sensory attributes, particularly visual cues, will influence choice to a lesser extent online than offline. This implies that marketers who rely strongly on visual cues to influence offline purchases of their brands, may be disappointed by the level of online sales that they are able to generate. Theirs study also suggests that brands can have more or less impact online than in traditional supermarkets de-pending on the extend of relevant information available for making choices in these markets.

Perter, Wilson and Davis [20] compare consumer brand loyalty in online and traditional shopping environments for over 100 brands in 19 grocery product categories. The result shows that observed brand loyalty for high market share brands bought online is significantly greater than expected, with the reverse result for small share brands. In contrast, in the traditional shopping environment, the difference between observed and predicted brand loyalty is not related to brand share.

\subsection{Gender Differences}

Gender is a very useful, yet private, piece of information that can help infer many important clues, such as customer shopping interests and user behaviors. A study by Yoo and Lauren [1] showed that male and female showed significant differences in their shopping orientations, online in-formation searches and purchase experiences; Soonyong Bae and Taesik [3] found that the effect of online consumer reviews on purchase intention is stronger for females than males. The negativity effect, that consumers are influenced by a negative review more than by a positive review, is also found to be more evident for females. From Shen Xuewu and Nie Guihua's experiment [45], we can conclude that males seem to exhibit more positive beliefs about Web advertising versus traditional media advertising, relative to females.

There are some other papers which have done research into the relation-ship between gender and shopping behavior. Yoo-Kyoung Seock and Lauren R. Bailey [1] investigated college students' shopping orientations, and examined the relationships between their shopping orientations and searches for information about and purchases of apparel products online and the differences between male and female students. They found that male and female participants showed significant differences in their shopping orientations, online information searches and purchase experiences. 
However, the limitation of this paper is that its research group is only college students, which leads to the lack of universality of its experimental results. Frank Ulbrich, Tina Christensen and Linda Stankus [2] approaches the question of whether on-line shopping preferences differ from a gender perspective by means of questionnaires. Our main catch from this paper is that significant differences might not show on the construct level but only when features are individually compared with each other. Based on this theory, we will first analyze individual features in the following experiments.

Through the above literature analysis, we can conclude that gender is an important factor affecting consumers' frequency of online shopping, probability to shop online and attitude towards online shopping. Re-searchers also found differences between men and women in their perceptions towards online shopping. Soonyong and Taesik [3] found that the effect of online consumer reviews on purchase intention is stronger for females than males and females are more easily influenced by a negative review. However, Ching Chen and Cheng $\mathrm{Hu}$ [16] pointed out that there are many interrelated antecedents that will influence customers' purchasing attitudes and behaviors in very complex ways.

Our approach differs from previous research by explicitly taking into account that males and females have different perceptions of what is considered important on offline shopping experience. First, we focus our research on brands and categories that are closely related to consumer behavior. In addition, we select frequent shoppers through data screening, making gender differences more persuasive. Finally, our experimental group is larger than the experimental population in other papers before, which means including all ages and professions.

\subsection{Gender Prediction}

Precisely because the complexity of the relationship between gender and behaviors, much research work has been done on gender prediction until now. As a result, gender prediction has been studied in different scenarios in academia. Early work on gender prediction attempted to predict gender based on the facial features, a person's face provides a lot of in-formation such as age, gender and identity. Michele, Liangliang and John [14] proposed a method to extract gender information from the pictures posted in social media feeds and achieved 88\% mean accuracy; Dileep M R and Ajit Danti [9] proposed an effective method named Multiple Hierarchical decision based on Neural Networks to predict human age and gender from facial images. Fake biometric identifiers can be of the form where one person imitates as another, so Anusree Bhaskar [10] presented a software based multi-biometric system to classify real and fake face samples and gender classification. With AR face database, Qingqing Lu, Jianfeng Lu and Dongjun Yu [11] build a convolutional neural network for gender classification based on facial image. These papers have proved the feasibility of classifying men and women by face images.

Beside the methods based on the analysis of facial features, recently, re-searchers focused on authorship studies, which are tasks of determining or predicting author characteristics by analyzing texts created by him/her. Text data in mobile devices are known to have high discriminative power for gender, Michael Crawford and Xingquan Zhu [15] proposed an approach for gender prediction using masked words as features and showed that gender prediction performance can be boosted by incorporating network topology statistics. Nesrine, Hassiba and Youcef [7] improved the gender classification based on offline handwritten text based on the use of local descriptors. The experiments above confirm that some methods through neural networks can effectively classify men and women by hand writing.

Recently, the fast development of online social networks and mobile computing technologies accumulated large scale of user data, making it possible and also valuable to infer users' demographic attributes in these scenarios. Thanks to the development of big data technology, more and more researchers have investigated the use of user behaviors on web applications to predict their demographic information. Do Viet Phuong and Tu Minh Phuong [21] investigated a number of features derived from browsing log data to predict users' gender and achieved a macro-averaged F1 score of 0.805. Different gender of users has different view on products, particularly in appreciation of fashion related products, the gender influence is much important. Qiufang Peng and Yang Liu [18] 
used seven characteristics chosen from online based e-commerce product browsing history data and used support vector machines set model by these seven characteristics to predict users' gender. By analyzing and training the model, this model achieved a $79.21 \%$ accuracy rate. Duc Duong, Hanh Tan and Son Pham [22] proposed a method for predicting the gender of customers based on their catalog viewing data on e-commerce systems, such as the date and time of access, list of categories and products viewed, etc. With employing a machine learning approach, this method results in a $81.2 \%$ accuracy rate and $81.4 \%$ on macro F1 score.

In the next section, we propose a framework to help us assess the relative impact of brand, prices and other search attributes on consumer choices of different genders. Using this framework, we predict the gender of a consumer by analyzing the purchase data of the consumer, aiming to provide important information for marketing, personalization, and user behavior research. We also compare the performance of different algorithms for gender prediction, thus providing a theoretical basis for further research in the future.

\section{Approach}

\subsection{System Overview}

In this work, we develop a framework which can take purchase data for users with known gender, extract features and class labels to create a training dataset. At the same time, we shall validate the experimental results through the test dataset.

\subsection{Features}

The feature set we used in this work can be divided into two types, which we call original features and external features.

1) Original features: Original features include temporal and individual brands/categories. Temporal features and features related to timestamp and frequency of purchasing activities. Individual brands/categories features consist of all categories and brands in the system. Because provided datasets contain all the categories and brands, we just extracted them and used as features. The contribution of brand features to offline gender prediction is unknown. Arvind and Wu [19] point out that brands can have more or less impact online than in traditional supermarkets depending on the extend of relevant information available for making choices in these markets. In addition to the two basic features of brand and category, for each consumer, we count the number of times the user has purchased and used that number as another unique feature which can be used as a dimension of evaluation system. Obviously, the more frequent the consumer purchases, the higher the accuracy of the gender classification is.

2) External features: Beside individual categories/ brands features, we hypothesize that some external features such as weather and name also reflect gender of the viewer. First of all, weather feature will affect the offline shopping form the visual terms. However, there are few scholars attend to study the relationship between weather and gender before, so we cannot conclude that weather feature is really helpful on gender prediction. Because of this, we will do further research on it through experiments. Second, lots of studies have proved that a simple and effective classification method for user gender prediction may be based on user name text: Wang Jingjing, Li Shoushan and Huang Lei [23] proposed a classifier fusion method to classify the users into male and female with the information provided by Chinese Micro-blog. Experimental result shows that in the gender classification of Chinese micro-blog, the text characters in user name text have certain classification performance. Burger et.al [24] used Twitter's tweets and personal data (account name, full name, personal description etc.) as well as their combination as features to identify the user's gender. Our study also uses the user's name as one of the features of the gender prediction experiment.

3) Fusion techniques: We divide the features into two kinds: original features and external features. In the following experiments, we will screen the most valuable features through experiments and fuse these features together. In the process of fusing features, we use a variety of 
fusion methods to compare their performance, so that we can see which fusion method is the best for offline gender prediction.

\section{Experimental Settings}

Here we introduce the experimental settings including the datasets, baseline methods, and evaluation metrics.

\subsection{Datasets}

We conducted experiments using real-world datasets. As mentioned earlier, the purchase data come from a large shopping mall in Beijing. Furthermore, the data used in this study span the 38 months of January 2014 through February 2017, during which the service was well established and had a reasonable customer base. Table I shows a summary of the datasets.

TABLE I. A SUMMARY OF THE DATASETS

\begin{tabular}{|c|c|}
\hline Interval & 2014.1.1-2017.2.21 \\
\hline Total Consumption Records & 437438 \\
\hline Total Members & 92194 \\
\hline Male Members & 31870 \\
\hline Female Members & 58556 \\
\hline Unknown Gender Members & 1768 \\
\hline Total Brand & 392 \\
\hline Total Category & 10 \\
\hline
\end{tabular}

The data was divided into training and test sets with the radio 4:1. A single log in the training data file is composed of four types of information:

- Buying time;

- Consumer name;

- Brand

- Category

- Human comfort index (ssd as short)

Human comfort index is a commonly used method to represent human comfort in daily life. It mainly depends on three indexes: air temperature, humidity and wind speed.

\subsection{Evaluation Metrics}

As evaluation metrics, macro F1 score and balanced accuracy measure were considered.

We judged the performance of the proposed and a baseline method in terms of precision $\mathrm{p}$, recall $\mathrm{r}$, and F1 score. For each class (male, female), precision is defined as the number of correctly predicted cases divided by the number of all predictions of this class. Recall is defined as the number of correctly predicted cases divided by the number of all cases of this class. F1 is the harmonic mean of precision and recall:

$$
\mathrm{F} 1=\frac{2 p r}{p+r}
$$

We also report macro-averaged F1 score, which is the average of F1 scores of two classes: male and female.

Beyond macro F1 score, the balanced accuracy measure (BAC) was also used to evaluate the model. Balanced accuracy is defined as an average accuracy obtained on either class and can avoid inflated performance estimates on imbalanced datasets.

$$
\text { balanced accuracy }=\frac{0.5 * t p}{t p+f n}+\frac{0.5 * t n}{t n+f p}
$$

Where $t p$ is true positives, $t n$ is true negatives, $f p$ is false positives, and $f n$ is false negatives. 


\section{Results and Discussion}

In order to evaluate the performance of original and external features, we conducted experiments on different sets of features, including original features and external features only and combination of both types of features. The training data and testing datasets are provided separately (the radio is 4:1). Therefore, our model was created based on the training dataset and tested on a different dataset. In addition, to verify the effects of purchase times, we conducted several experiments in the dimension of the number of times the consumer purchased. Table II shows the number of people under different buying times.

\section{table il. Number of PeOPle Under Different Buying Times}

\begin{tabular}{|c|c|}
\hline Buying Times & Number \\
\hline$>=0$ & 87852 \\
\hline$>=1$ & 57626 \\
\hline$>=2$ & 43638 \\
\hline$>=3$ & 34399 \\
\hline$>=4$ & 27812 \\
\hline$>=5$ & 22811 \\
\hline$>=6$ & 19171 \\
\hline$>=7$ & 16302 \\
\hline$>=8$ & 14086 \\
\hline
\end{tabular}

We first carried out experiments based on single feature. With regard to category, brand and weather feature, we first established feature vectors, and then calculated the accuracy of gender prediction by means of KNN. Among them, because the total number of brands in the data set is really abundant, we selected 107 brands with the chi square value greater than 5.99 , and then conduct PCA to reduce the dimension. Through the feature reduction experiment, we found that the accuracy is the highest when the 392 dimension is reduced to 8. The following table shows the accuracy of gender prediction using different features with the number of consumers consumed in the dataset.

TABLE III. RESUlTS OF EXPERIMENTS WITH SiNGLE FEATURE

\begin{tabular}{|c|c|c|c|c|}
\hline \multirow{2}{*}{ Buying Times } & \multicolumn{4}{|c|}{ Feature } \\
\cline { 2 - 5 } & ssd & category & brand & name \\
\hline$>=0$ & 61.9 & 60.8 & 59.2 & 71.2 \\
\hline$>=1$ & 60.0 & 60.7 & 62.3 & 70.6 \\
\hline$>=2$ & 62.0 & 62.4 & 63.7 & 71.6 \\
\hline$>=3$ & 63.0 & 62.6 & 64.5 & 72.5 \\
\hline$>=4$ & 63.0 & 63.5 & 65.8 & 73.0 \\
\hline$>=5$ & 64.4 & 63.8 & 65.7 & 73.3 \\
\hline$>=6$ & 64.5 & 64.8 & 66.6 & 73.6 \\
\hline$>=7$ & 65.2 & 65.3 & 66.5 & 73.8 \\
\hline$>=8$ & 65.2 & 65.7 & 66.7 & 74.0 \\
\hline
\end{tabular}

According to Table III, we found that the more consumer purchased the higher the accuracy rate achieved. This is in line with expectations because the more times consumers purchase, the more accurate we are to predict their gender through their purchase data. In order to simplify the experimental process, we followed the experiment with consumers who consumed more than 8. At the same time, the experimental results also show that if only one feature is allowed, the gender prediction using the name feature will achieve the best effect. However, the accuracy of all experiments until now is below seventy percent, which to some extend shows that only one feature cannot meet the experimental expectations, so the experiment of feature fusion is urgent.

The effect of combination of features was tested using the popular machine learning methods, namely Random Forest, Bagging, and Bayes Net. In order to further verify the contribution of each feature to gender prediction, we carried out the following fusion experiments: fusion of weather 
features and brand features, fusion of brand features and name features as well as fusion of weather features, brand features and name features. We didn't conduct fusion experiments with weather features and name features, because in that case the predictions do not relate to the users' consumer behavior and cannot not be used in practice. Table IV-V show the results of fusion experiments (buying time $>=8$ ):

TABLE IV. RESULTS OF EXPERIMENTS WITH FUSION USING GAUSSIAN NAÏVE BAYES

\begin{tabular}{|l|c|c|c|}
\hline \multirow{2}{*}{$\begin{array}{c}\text { Evaluatio } \\
\text { n Metrics }\end{array}$} & \multicolumn{3}{|c|}{ Fusion Features } \\
\cline { 2 - 4 } & $\begin{array}{c}\text { Ssd \& } \\
\text { brand }\end{array}$ & $\begin{array}{c}\text { Brand \& } \\
\text { name }\end{array}$ & $\begin{array}{c}\text { Ssd \& brand \& } \\
\text { name }\end{array}$ \\
\hline accuracy & 67.3 & 74.0 & 80.0 \\
\hline Macro F1 & 51.3 & 73.3 & 78.5 \\
\hline BAC & 53.0 & 79.2 & 81.1 \\
\hline
\end{tabular}

TABle V. Results of EXPERIMENTS By DifFERENT Fusion Methods

\begin{tabular}{|l|c|c|c|}
\hline \multirow{2}{*}{$\begin{array}{c}\text { Evaluatio } \\
\text { n Metrics }\end{array}$} & \multicolumn{3}{|c|}{ Fusion Methods } \\
\cline { 2 - 4 } & $\begin{array}{c}\text { Gaussian Naïve } \\
\text { Bayes }\end{array}$ & Bagging & $\begin{array}{c}\text { Random } \\
\text { Forests }\end{array}$ \\
\hline accuracy & 80.0 & 86.8 & 96.2 \\
\hline Macro F1 & 78.5 & 84.2 & 95.4 \\
\hline BAC & 81.1 & 83.7 & 95.6 \\
\hline
\end{tabular}

Based on the experimental results of fusion experiments, we can draw the following conclusions:

Firstly, the external features when combining with original features remarkably improve prediction result compared with using original features or external features only.

Secondly, as the results shown in Table V, Random Forest achieved the best results while Bayes Net gave the lowest performance on both BAC and Macro F1 score.

Lastly, experimental studies with real data show that our method outperformed a baseline method by a large margin. This implementation is not limited to a single database, but also can be applied to different database and also the datasets which can be downloaded from the internet. Moreover, we believe that the improvement will increase more when applying our method on more realistic datasets.

\section{Conclusion and Future Work}

In this study, we investigated a method for predicting the gender of customer based on theirs purchase data and some external factors. Experimental results show that this feature design is work best on the Random Forest algorithm with supporting techniques such as Principal Component Analysis, Chi-square Parameter Testing to deal with dimension reduction problem and feature selection problem. In addition, the advantage of method is that it can be easily applied to other datasets because it uses no dataset-specific features.

To sum up, the contributions of the paper are summarized into three points:

Firstly, demographic attributes such as gender of consumers provide important information for marketing, personalization, and user behavior research; Experimental results show that weather, a feature that few scholars have paid attention to before, has an impact on the gender prediction of offline purchase data. Our next work will also examine the relationship between weather feature and shopping behavior among different gender consumers.

Secondly, we can see that gender prediction offline has significant differences from online gender classification. Compared with the online data, offline data lacks user behavior related data (browse, click, cancel orders, etc.), so the gender prediction task in traditional shopping environments needs to be assisted by some external data, such as weather, consumers' name and so on.

Lastly, from the experiments conducted on real-world dataset acquired from a large shopping 
mall in Beijing, the potential of the proposed framework was confirmed, and the trade-offs between diverse parameters and performance evaluation measures were observed. Furthermore, although we experimented only with gender prediction, the method can be extended to predict other demographic information such as age.

Additional research is needed to enhance the validity of this research. First, Research should delve more into the problem of how to make gender-specific differences more visible on the construct level, because it can create more valuable insights into which aspects are important to offline shoppers. Secondly, future research should be designed to eliminate some of the shortcomings of this study, extract more useful information from the relation between categories/brands purchased during a single purchasing behavior. Last, we plan to extend our work to predict other user attributes such as age, user interests and preferences from user generated multi-media content. This is deemed important because it can provide rich and valuable information for customized marketing and personalized recommendation systems.

\section{References}

[1] Yookyoung, S., \& Laurenr, B. (2008). The influence of coullege students' shopping orientations and gender differences on online information searches and purchase behaviours. International Journal of Consumer Studies, 32(2), 113-121.

[2] Ulbrich, F., Christensen, T., \& Stankus, L. (2011). Gender-specific on-line shopping preferences. Electronic Commerce Research, 11(2), 181-199.

[3] Bae, S., \& Lee, T. (2011). Gender differences in consumers' perception of online consumer reviews. Kluwer Academic Publishers.

[4] Babakus, E., \& Yavas, U. (2008). Does customer sex influence the relationship between perceived quality and share of wallet ?. Journal of Business Research, 61(9), 974-981.

[5] Mirza, A., Moetesum, M., Siddiqi, I., \& Djeddi, C. (2017). Gender Classification from Offline Handwriting Images Using Textural Features. International Conference on Frontiers in Handwriting Recognition(pp.395-398). IEEE.

[6] Bouadjenek, N., Nemmour, H., \& Chibani, Y. (2015). Histogram of Oriented Gradients for writer's gender, handedness and age prediction. International Symposium on Innovations in Intelligent Systems and Applications (pp.1-5). IEEE.

[7] Bouadjenek, N., Nemmour, H., \& Chibani, Y. (2015). Local descriptors to improve off-line handwriting-based gender prediction. Soft Computing and Pattern Recognition (pp.43-47). IEEE.

[8] Erbilek, M., Fairhurst, M., \& Li, C. (2016). Exploring gender prediction from digital handwriting. Signal Processing and Communication Application Conference (pp.789-792).

[9] Dileep, M. R., \& Danti, A. (2016). Multiple hierarchical decision on neural network to predict human age and gender. International Conference on Emerging Trends in Engineering, Technology and Science (pp.1-6).

[10] Bhaskar, A., \& Aneesh, R. P. (2015). Advanced algorithm for gender prediction with image quality assessment. International Conference on Advances in Computing, Communications and Informatics (pp.1848-1855). IEEE.

[11] Lu, Q., Lu, J., \& Yu, D. (2015). Gender classification based on the convolutional neural network. Intelligent Control and Automation (Vol.54, pp.1962-1965). IEEE. (in Chinese).

[12] Peisong Zhu, Tieyun Qian, Ming Zhong, \& Xuhui Li. (2016). Inferring users’ gender from interests: a tag embedding approach.

[13] Aravantinou, C., Simaki, V., Mporas, I., \& Megalooikonomou, V. (2015). Gender Classification of Web Authors Using Feature Selection and Language Models. International 
Conference on Speech and Computer, Speecom (Vol.47, pp.128-128).

[14] Merler, M., Cao, L., \& Smith, J. R. (2015). You are what you tweet...pic! gender prediction based on semantic analysis of social media images. IEEE International Conference on Multimedia and Expo (Vol.107, pp.1-6). IEEE.

[15] Crawford, M., \& Zhu, X. (2015). Gender Prediction in Random Chat Networks Using Topological Network Structures and Masked Content. IEEE International Conference on Information Reuse and Integration(pp.174-181). IEEE Computer Society.

[16] Kuruvilla, S. J., Joshi, N., \& Shah, N. (2010). Do men and women really shop differently? an exploration of gender differences in mall shopping in india. International Journal of Consumer Studies, 33(6), 715-723.

[17] Wang, P., Guo, J., Lan, Y., Xu, J., \& Cheng, X. (2016). Your Cart tells You: Inferring Demographic Attributes from Purchase Data. ACM International Conference on Web Search and Data Mining (Vol.1, pp.173-182). ACM.

[18] Qiufang Pang, \& Yang Liu. (2016). Research of gender prediction based on SVM with Ecommerce data. Journal of Shandong University (Natural Science), 51(07), 74-80. (in Chinese).

[19] Degeratu, A. M., Rangaswamy, A., \& Wu, J. (2000). Consumer choice behavior in online and traditional supermarkets: the effects of brand name, price, and other search attributes. International Journal of Research in Marketing, 17(1), 55-78.

[20] Phuong, D. V., \& Tu, M. P. (2014). Gender Prediction Using Browsing History. Knowledge and Systems Engineering. Springer International Publishing.

[21] Duong, D., Tan, H., \& Pham, S. (2016). Customer gender prediction based on E-commerce data. Eighth International Conference on Knowledge and Systems Engineering (pp.91-95). IEEE.

[22] Jingjing Wang, Shoushan Li, \& Lei Huang. (2014). User Gender Classification in Chinese Micro-blog. Journal of Chinese Information Processing, 28(06), 150-155. (in Chinese)

[23] Burger, J. D., Henderson, J., Kim, G., \& Zarrella, G. (2011). Discriminating gender on Twitter. Conference on Empirical Methods in Natural Language Processing (pp.1301-1309). Association for Computational Linguistics.

[24] Hernández, B., Jiménez, J., \& Martín, M. J. (2011). Age, gender and income: do they really moderate online shopping behaviour?. Online Information Review, 35(1), 113-133.

[25] Rodolico, N. (2015). E-Commerce Purchase Intention in Emerging Markets: The Influence of Gender and Culture. Cross-Cultural Design Applications in Mobile Interaction, Education, Health, Transport and Cultural Heritage. Springer International Publishing.

[26] Scheers, L. V. (2007). The effect of consumer price knowledge and gender on retail marketing strategy. African Journal of Business Management, 1(4), 92-98.

[27] Chen, I. C., \& Hu, S. C. (2012). Gender differences in shoppers' behavioural reactions to ultra-low price tags at online merchants. Electronic Commerce Research, 12(4), 485-504.

[28] Piyush Sharma, Ivy S.N. Chen, \& Sherriff T.K. Luk. (2012). Gender and age as moderators in the service evaluation process. Journal of Services Marketing, 369(2), 20120464-20120464.

[29] Ellis, L., Abild, M. L., Buker, H., Park, J. R., \& Ping, H. (2012). Gendered shopping: a seven country comparison. Mankind Quarterly, 52(3-4), 336-357.

[30] Tan, T. M., Tan, S. M., Lee, W., Bee, M., Fang, O., \& Liew, T. W. . Does gender contribute moderating effect in brand equity model?.

[31] Pei Ling Lim, \& Dr. Rashad Yazdanifard. (2014). Does gender play a role in online consumer behavior?. , volume 14, pp. 61-68. 
[32] Akhlaq, A., \& Ahmed, E. (2016). Gender differences among online shopping factors in pakistan. Organizations \& Markets in Emerging Economies, 7.

[33] Hou, J., \& Elliott, K. (2016). Gender differences in online auctions. Elsevier Science Publishers B. V.

[34] Liu, W. Y., Lin, C. C., Yang, S. L., \& Deng, D. J. (2013). On gender differences in consumer behavior for online financial transaction of cosmetics. Mathematical \& Computer Modelling, 58(1-2), 238-253.

[35] Jansen, B. J., Moore, K., \& Carman, S. (2013). Evaluating the performance of demographic targeting using gender in sponsored search. Information Processing \& Management, 49(1), 286-302.

[36] Raajpoot, N. A., Sharma, A., \& Chebat, J. C. (2008). The role of gender and work status in shopping center patronage. Journal of Business Research, 61(8), 825-833.

[37] Lian, J. W., \& Yen, D. C. (2014). Online shopping drivers and barriers for older adults: age and gender differences. Computers in Human Behavior,37(37), 133-143. 